\title{
A Compact Electronic Speckle Pattern Interferometry System using a Photopolymer Reflection Holographic Optical Element
}

\author{
Guntaka Tulasi Sridhar Reddy \\ Technological University Dublin \\ Raghavendra Jallapuram \\ Technological University Dublin \\ Vincent Toal \\ Technological University Dublin, vincent.toal@tudublin.ie
}

See next page for additional authors

Follow this and additional works at: https://arrow.tudublin.ie/cieocon2

Part of the Optics Commons

\section{Recommended Citation}

Reddy, S. et al. (2005) A compact electronic speckle pattern interferometry system using a photopolymer reflection holographic optical element. SPIE proceedings of 17th International Conference on Photonics in Europe, V.5856, 157. doi.org/10.21427/k9zg-dw31

This Conference Paper is brought to you for free and open access by the Centre for Industrial and Engineering Optics at ARROW@TU Dublin. It has been accepted for inclusion in Conference Papers by an authorized administrator of ARROW@TU Dublin. For more information, please contact arrow.admin@tudublin.ie, aisling.coyne@tudublin.ie, gerard.connolly@tudublin.ie.

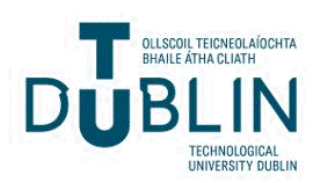




\section{Authors}

Guntaka Tulasi Sridhar Reddy, Raghavendra Jallapuram, Vincent Toal, Izabela Naydenova, Suzanne Martin, and Svetlana Mintova 
2005-01-01

\section{A compact electronic speckle pattern interferometry system using a photopolymer reflection holographic optical element}

Sridhar Reddy

Dublin Institute of Technology

Raghavendra Jallapuram

Dublin Institute of Technology

Vincent Toal

Dublin Institute of Technology, vincent.toal@dit.ie

Izabela Naydenova

Dublin Institute of Technology, izabela.naydenova@dit.ie

Suzanne Martin

Dublin Institute of Technology, suzanne.martin@dit.ie

See next page for additional authors

\section{Recommended Citation}

S. Reddy Guntaka, J. Raghavendra, V. Toal, I. Naydenova, S. Martin, S. Mintova, A compact electronic speckle pattern interferometry system using a photopolymer reflection holographic optical element, SPIE proceedings of 17th International Conference on

Photonics in Europe, V.5856, 157, 2005 
Authors

Sridhar Reddy, Raghavendra Jallapuram, Vincent Toal, Izabela Naydenova, Suzanne Martin, and Svetlana Mintova 


\title{
A compact electronic speckle pattern interferometry system using a photopolymer reflection holographic optical element
}

Sridhar Reddy Guntaka ${ }^{\mathrm{a}}$, J.Raghavendra ${ }^{\mathrm{a}}$, Vincent Toal ${ }^{\mathrm{a}}$, Izabela Naydenova ${ }^{\mathrm{a}}$, Suzanne Martin ${ }^{\mathrm{a}}$ S.Mintova ${ }^{\mathrm{b}}$

\author{
${ }^{a}$ Centre for Industrial \& Engineering Optics, Dublin Institute of Technology, School of Physics, \\ Kevin Street, Dublin-8, Ireland. \\ ${ }^{\mathrm{b}}$ Department of chemistry, University of Munich, 81377 Munich, Germany
}

\begin{abstract}
A simple and compact electronic speckle pattern interferometry system using a reflection holographic optical element is presented. The reflection holographic optical element is recorded on an acrylamide based photopolymer formulated and prepared at the Centre for Industrial \& Engineering Optics. Light intensity of $40 \mathrm{~mW} / \mathrm{cm}^{2}$ with an exposure time of 60 seconds was used in fabricating the holographic optical element. The vibration mode patterns of a $4 \mathrm{~cm}$ diameter thin circular sheet of brass metal attached to a $4 \mathrm{~cm}$ diameter paper cone loud speaker are presented.
\end{abstract}

Key words: ESPI, Photopolymer, Vibration modes, HOE, reflection hologram.

\section{Introduction}

Electronic Speckle Pattern Interferometry (ESPI) is one of several promising optical non contact, whole field laser techniques available for measuring surface displacements. It is a well established non destructive evaluation tool used in optical metrology applications. It utilizes the speckle pattern produced by an optically rough surface when illuminated by laser light. ESPI is also known as; digital holography, electronic holography and TV holography ${ }^{1}$. Its development as an experimental technique originates with Holographic Interferometry (HI). Laser speckle noise was regarded as the bane of holography ${ }^{2}$, but the speckle phenomenon became the stepping stone for the speckle metrology. Butters and Leendertz developed the technique in $1970^{3}$, since when it has been used in a variety of engineering and other applications ${ }^{4}$. A system was demonstrated using the vidicon based TV camera with a standard frame rate of 25 frames per second. Deformation of the order of wavelength of light used can be extracted using the ESPI technique. The advantage of no processing of recording materials and minimal requirements for accuracy of optical alignment allows ESPI to surpass Holographic Interferometry (HI). In recent years ESPI has been used for industrial applications with the introduction of new lasers and optical technology. ESPI is also used to study the small amplitude vibration mode patterns. By applying phase shifting techniques in an ESPI system the complete whole field displacement map can be obtained.

\section{ESPI system-Fringe formation}

The basic ESPI system consists of an optical head, CCD camera and a host computer with an image processing system. An object surface illuminated with laser light produces a speckle pattern in the reflected light, which is imaged on to the CCD camera. A uniform or a speckled reference wave is also allowed to fall on the image plane. The resultant speckle interferogram is stored and displayed on the television monitor (Frame 1). Deformation of the test object produces a path difference between the light scattered from the object surface and the reference wave and the speckle interferogram is modified (Frame 2). The modified speckle pattern is either subtracted or added to the previously stored speckle pattern (Frame $1 \pm$ Frame 2). Usually addition fringe formation is associated with pulsed laser illumination rather than with $\mathrm{CW}$ laser illumination. The resultant signal is rectified and displayed on the monitor. The bright and dark fringes displayed on the monitor are the correlation fringes and they represent the contours of constant displacement.

The complex amplitude of the intensity distribution of the interference pattern in the image plane before the object deformation is given by ${ }^{5}$ 


$$
I_{\text {Undisplaced }}=I_{1}+I_{2}+2 \sqrt{I_{1} I_{2}} \cos (\psi)
$$

Where $\psi$ is the phase difference between the object wave front and the reference wave and $I_{1}$ and $I_{2}$ are the intensities of the two waves.

If the object undergoes a static displacement, a phase change of $\Delta \phi$ is introduced between the two waves. The intensity distribution is now given by

$$
I_{\text {displaced }}=I_{1}+I_{2}+2 \sqrt{I_{1} I_{2}} \cos (\psi+\Delta \phi)
$$

The subtracted signal $V_{S}$ is given by

$$
4 \sqrt{I_{1} I_{2}} \sin \left(\psi+\frac{\Delta \phi}{2}\right) \sin \left(\frac{\Delta \phi}{2}\right)
$$

This signal contains both positive and negative values. The negative signal is displayed on the monitor as an area of blackness. To avoid loss of signal the subtracted signal is rectified before displaying on the monitor. Brightness on the monitor is given by

$$
B=4 K\left[I_{1} I_{2} \sin ^{2}\left(\psi+\frac{\Delta \phi}{2}\right) \sin ^{2}\left(\frac{\Delta \phi}{2}\right)\right]^{\frac{1}{2}}
$$

If the brightness is averaged along a line of constant $\Delta \phi$, it varies between $\mathrm{B}_{\max }$ and $\mathrm{B}_{\min }$ and the values are given by

$$
\begin{aligned}
& B_{\max }=2 K \sqrt{I_{1} I_{2}} \text { when } \Delta \phi=(2 n+1) \pi \text { with } \mathrm{n}=0,1,2 \\
& B_{\text {min }}=0 \text { when } \Delta \phi=2 n \pi \text { with } \mathrm{n}=0,1,2 .
\end{aligned}
$$

The dark and bright fringes are displayed on the TV monitor.

\section{Conventional ESPI systems}

The optical head of an ESPI system can be constructed in different ways depending on the type of measurement required. The optical systems can be configured in such a way that they can measure both the in plane and out of plane displacements ${ }^{5}$. Conventional systems are built using optical elements including lenses, mirrors, beam splitters and beam combiners. A complete designed system consists of the optical hardware, test object, CCD camera, laser source and a PC with frame grabber. Alignment of optics in such a system is critical to obtain good quality interferograms. A schematic diagram of an out of plane sensitive conventional ESPI system is shown in Fig1. Alignment difficulties are minimized by constructing a miniaturized ESPI system by replacing the optical hardware in a conventional ESPI system with a holographic optical element (HOE). ESPI systems with HOEs fabricated using silver halide plates or thermo plastic recording material have been reported ${ }^{6}$. A transmission acrylamide based photopolymer HOE was used in an ESPI system for strain measurement ${ }^{7,8}$. A holographically reconstructed master object was used in comparative speckle interferometry ${ }^{9}$. A holographic parabolic mirror recorded on HP series silver halide emulsions was used as the receiver for infrared optical communications. ${ }^{10,11}$ The HP series silver halide emulsions have been successfully used in an ESPI system and the results are yet to published else where. 


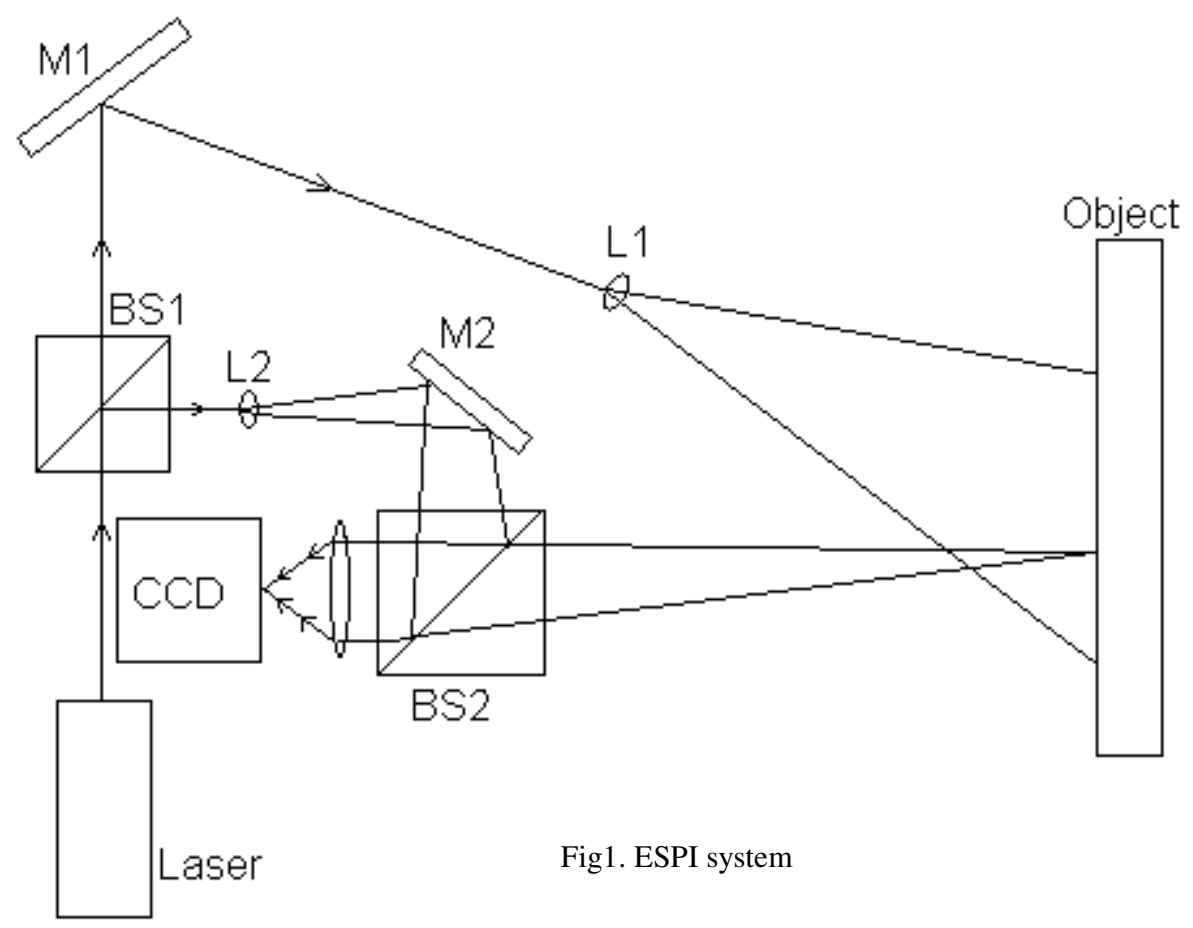

\section{Reflection HOE based system}

Using a reflection HOE makes the system simpler than the system with a transmission HOE, also recording and reconstruction stages for fabricating and using the transmission HOEs require more optics than with fabricating a reflection HOE. The experimental layout of a reflection HOE based ESPI system is shown in fig.2. The diverging beam from the laser illuminates the HOE and the CCD camera is placed in front of HOE to capture the reconstructed image; at the same time the camera sees the test object through the HOE. The path length imbalance in the interferometer can be altered by simply changing the distance between the test object and HOE. The intensity of object and reference beams can be made equal by rotating the HOE off Bragg angle with reference to its vertical and horizontal axis. This facilitates obtaining good quality interferograms.

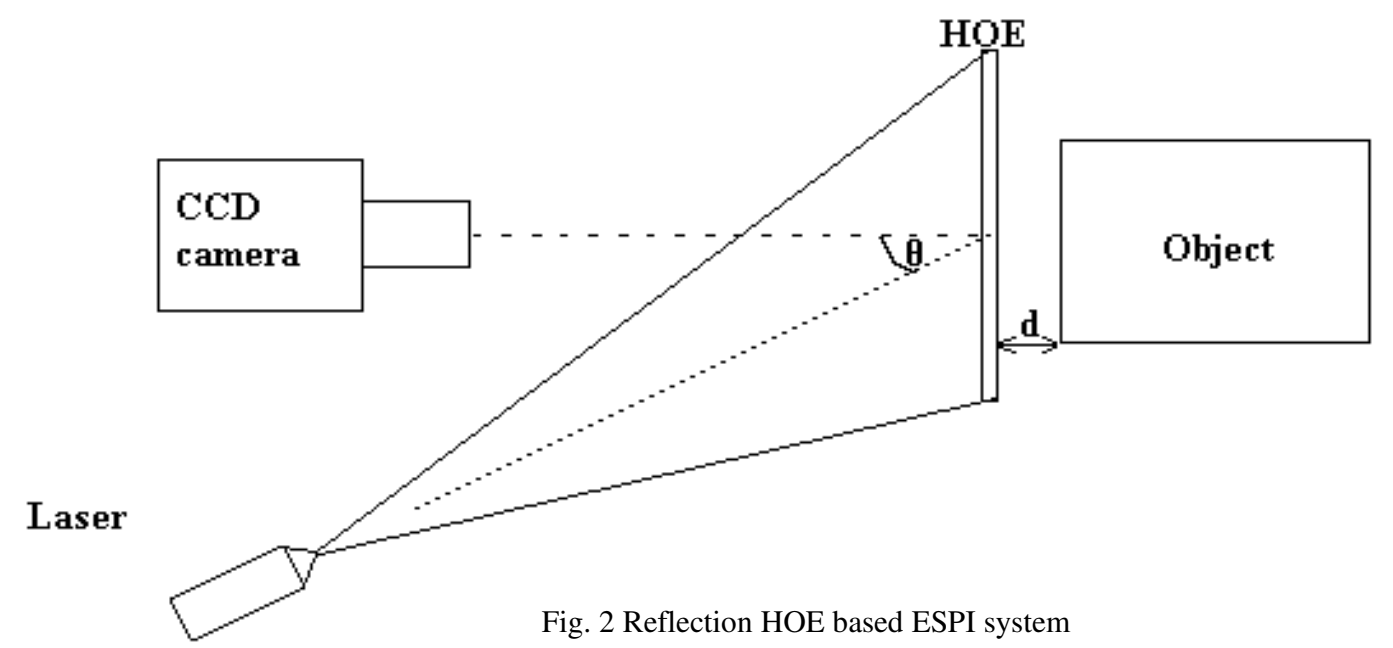




\section{Photopolymer for Fabricating HOEs}

There are several different recording media available for producing holograms. The commonly available hologram recording medium, silver halide plates requires, extensive chemical processing in the dark room. The ease of preparation, self processing capability of photopolymerizable recording medium has made a significant contribution to holography. The photopolymer recording medium consists of polymerizable monomer, dye, electron donor and binder. Photopolymers are very useful in HI due to their self processing capability. The acrylamide based photopolymer recording material has already been used in strain measurements using live fringe HI. ${ }^{12}$. Research at the Centre for Industrial \& Engineering Optics is in progress to characterize acrylamide based photopolymer as a potential holographic data storage medium. The material has good spatial frequency response for recording transmission holograms. Researchers have attained efficient gratings up to spatial frequencies of $3500 \mathrm{lines} / \mathrm{mm}^{13,14}$. In recent months the material's diffraction efficiency was improved in reflection mode. Self processing ability of the material allows reconstructing the holographic image instantaneously after the recording step. It also allows using the hologram as an optical element in an ESPI system.

\section{ESPI for vibration measurements}

ESPI system using a reflection HOE recorded in acrylamide based photopolymer was used to identify the mode patterns of an object when it is vibrating. Time averaged ESPI has numerous applications; including the automobile and aerospace industries ${ }^{15}$. The basic concept in time average ESPI is that a sinusoidally vibrating object will phase modulate light reflected from it with the modulation depth proportional to the vibration amplitude. The nodal areas, the areas not in motion, all will yield high definition speckle and the adjacent areas which are in motion will produce a speckle pattern that varies rapidly in phase. The intensity distribution of the time averaged interference pattern at the image plane of the CCD camera is 16

$I_{1}+I_{2}+4 I_{1} I_{2} J_{0}^{2}\left(\frac{4 \pi d_{0}}{\lambda}\right)$

Where $d_{0}$ is vibration amplitude of the object, $\mathrm{J}_{0}$ is the Bessel function of zero order. $\lambda$ is the wave length of the laser. The amplitude of vibration of the object can be extracted from the Bessel function. In the speckle fringe pattern the areas with maximum brightness (nodal areas) represent zero amplitude of vibration. Extracting the amplitude and phase of the vibration is only possible using phase shifting methods. Phase shift in an interferometer can be introduced by different methods, such as moving a mirror, tilting a glass plate, moving a grating, rotating a quarter wave plate or using an acousto-optic or electro-optic modulator ${ }^{16}$.

\subsection{Recording HOEs}

\section{Experimental}

Reflection HOEs were recorded in acrylamide based photopolymer layers using a $\mathrm{Nd}-\mathrm{YVO}_{4}$ laser at $532 \mathrm{~nm}$. The geometry for recording the HOE on a Newport vibration isolation table is shown in fig.3. The laser is switched on and allowed to run for 1 hour before recording the hologram, which enables the laser to be thermally stabilised. A hologram of a thick aluminium plate was recorded with the plate placed $1 \mathrm{~cm}$ apart from the recording medium.

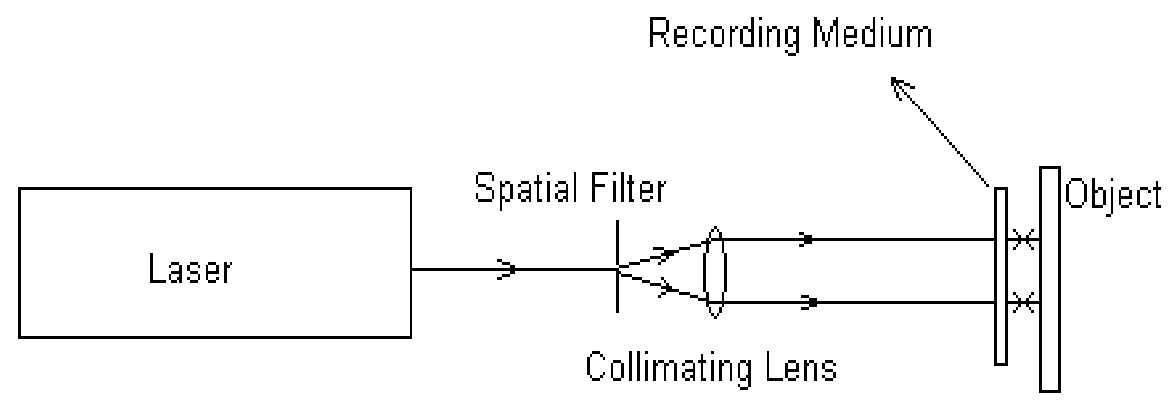

Fig. 3 HOE recording geometry 


\subsection{Vibration mode patterns with ESPI}

The vibration mode patterns of a thin circular sheet $4 \mathrm{~cm}$ diameter attached to a paper cone loud speaker $4 \mathrm{~cm}$ diameter were studied. The HOE is mounted on a rotational stage and illuminated by laser light. The HOE is rotated and positioned in such a way that a bright reconstructed image of the original object is imaged with CCD camera. The test object is placed behind the HOE. The CCD camera views the test object through the HOE. The vibration mode patterns at frequencies $1000 \mathrm{~Hz}, 2600 \mathrm{~Hz}, 6400 \mathrm{~Hz}$ are shown in fig. 4 .

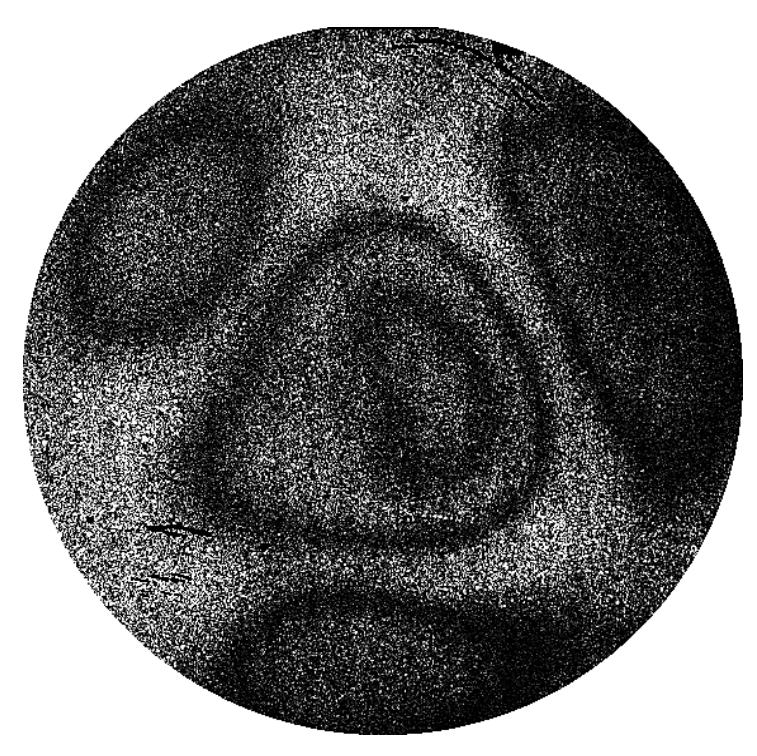

$1000 \mathrm{~Hz}$

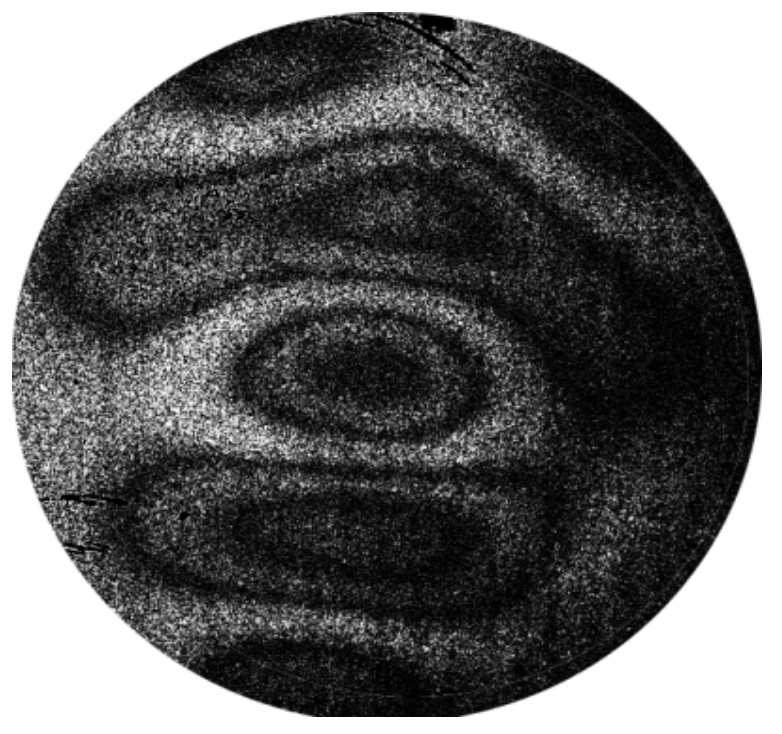

$2600 \mathrm{~Hz}$

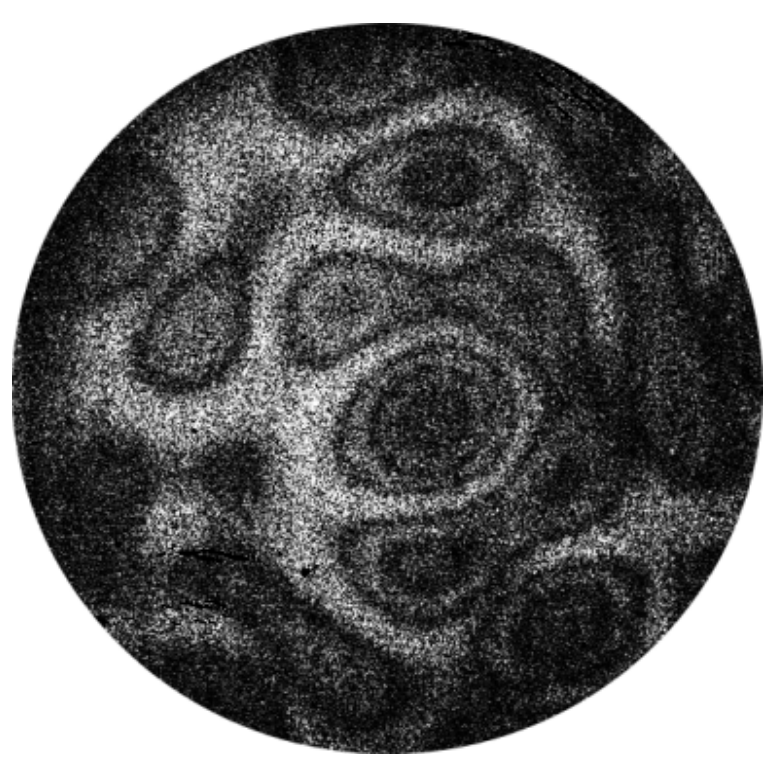

$6400 \mathrm{~Hz}$

Fig. 4 Vibration mode patterns 


\section{Future scope of work}

The complete displacement map of the object deformation can be obtained by implementing phase shifting methods. Phase shifting in the system using a reflection HOE can be implemented by drive current modulation of a laser diode. The drive current modulation introduces a phase change in the interferometer by modulating the wavelength. Complete displacement map of the object deformation can be obtained by using a visible diode laser which can be modulated and the same laser can be used to record the HOE and for phase shifting ESPI system.

\section{Acknowledgements}

The authors would like to acknowledge FOCAS for providing excellent laboratory facilities. FOCAS is funded under the Irish government National Development Plan 2002-2006 with assistance from European regional development fund.

\section{References}

1. Angel F Doval, “A systematic approach to TV holography,” Meas.Sci.Technol 11, R1-R36, (2000).

2. D.Gabor, "Laser speckle and its elimination," IBM.J.Rev.Develop, 14, 509-514, (1970).

3. J.N.Butters, J.A.Leendertz, "A double exposure technique for speckle pattern interferometry," Journal of Physics E: Sci. Instrum, 4, 277-279, (1971)

4. Nandigana Krishna Mohan, Pramod Rastogi "Recent developments in digital speckle pattern interferometry," Optics and Lasers in Engineering, 40, 439-445, 2003

5. R. Jones, C.Wykes, "Holographic and speckle interferometry," Cambridge university press, Cambridge, (1983)

6. V.Petrov, B.Lau, "Electronic speckle pattern interferometry with a holographically generated reference wave," Opt. Eng. 35, 2363-2370, (1996)

7. Sridhar Reddy Guntaka, Vincent Toal, Suzanne Martin, "Holographically recorded photopolymer diffractive optical element for holographic and electronic speckle pattern interferometry," App Opt, 41, 7475-7479, (2002).

8. S.R.Guntaka, V.Toal, S.Martin, "Holographic and Electronic Speckle Pattern Interferometry using a Photopolymer Recording Material," Strain, 40, 79-81, (2004)

9. L. Laszlo, Z. Fuzessy, J.Kornis, F.Gyimesi, “Comparative measurement by speckle pattern interferometry using holographically reconstructed object," Opt. Eng. 36, 3323-3326, (1997)

10. S.Jikova, M.Kavehard, "Holographic optical receiver front-end for wireless infrared indoor communications," Appl Opt, 40, 2828-2835, (2001).

11. S. Jivkova, S. Shurulinkov, M. Kavehrad, "Holographic Parabolic Mirror as a Receiver Optical Front End for Wireless Infrared Communications: Experimental Study," Appl Opt, 41(28), 5860-5865, (2002)

12. E.Mihaylova, B.Potelon, S.Reddy, V.Toal and C.Smith, "Mechanical characterization of unplasticised polyvinyl chloride thick pipes by optical methods," Optics and Lasers in Engineering, 41, 889-900, (2004).

13. Martin. S, Feely. C.A, Toal. V, "Holographic Characteristics of an Acrylamide Based Recording Material," Appl Opt, 36, 5757-5769, (1997).

14. I.Naydenova, J.Raghavendra, S.Martin, R.Howard, V.Toal, Suzanne Martin "Investigation of the diffusion process in self processing acrylamide based photopolymer system," App. Opt, 43, 2900-2905, (2004).

15. F.Chen, T.E.Allen, "Digital speckle pattern interferometry: some developments and applications for vibration measurement in the automotive industry," Opt. Eng 37, 1390-1397, (1998).

16. P.K.Rastogi, "Digital speckle pattern interferometry and related techniques," chapter4, John Wiley \& Sons Ltd, England, (2001). 